\title{
Subwavelength resolution of the annular photonic crystal with negative refraction
}

\section{F. Xia}

M. Yun

mjyun@qdu.edu.cn

M. Liu

J. Liang

W. Kong

Y. Wan
Key Laboratory of Photonics Materials and Technology, College of Physics Science, Qingdao University, Qingdao, 266071, China

Key Laboratory of Photonics Materials and Technology, College of Physics Science, Qingdao University, Qingdao, 266071, China

Key Laboratory of Photonics Materials and Technology, College of Physics Science, Qingdao University, Qingdao, 266071, China

Key Laboratory of Photonics Materials and Technology, College of Physics Science, Qingdao University, Qingdao, 266071, China

Key Laboratory of Photonics Materials and Technology, College of Physics Science, Qingdao University, Qingdao, 266071, China

Key Laboratory of Photonics Materials and Technology, College of Physics Science, Qingdao University, Qingdao, 266071, China

In this paper, negative refraction in the two-dimensional (2D) hexagonal lattices annular photonic crystal (APC) was theoretically studied. The annular photonic crystal was obtained by introduced circular-air-hole in the core of the Si-rod. The properties of the designed APC, such as photonic band structure, equal-frequency contours (EFCS) and the electric field distribution are analyzed by using Plane Wave Expansion (PWE) method and Finite-Difference Time-Domain (FDTD) method. Numerical simulations show that negative refraction and super-lens imaging can be realized in the designed annular photonic crystal for the normalized frequency from $0.2983(2 \pi c / a)$ to $0.347(2 \pi c / a)$. And it was also found that the resolution decreases linearly with the increasing of the inner radius.

[DOI: http://dx.doi.org/10.2971/jeos.2012.12045]

Keywords: Negative refraction, annual photonic crystal (APC), plane wave expansion (PWE), finite-difference time-domain (FDTD), superlens

\section{INTRODUCTION}

In 1968, Veselago first pointed out that the negative refraction phenomenon could be observed in the left-handed-materials (LHMs) which have the negative dielectric permittivity and negative magnetic permeability [1], Since then LHMs have attracted a lot of attention. Pendry proposed that a perfect lens could be realized by using the LHMs to reconstruct a perfect image [2]. It was known that the negative refraction can be realized in photonic crystal (PhC) [3]-[5]. In 1987, Yablonovitch and John proposed the concept of PhC independently. In recent years, theoretical and experimental results indicate that negative refraction phenomenon can be realized in the first band near the Brillouin zone center. This can be well explained by analyzing the equal-frequency contours (EFCs) of the first band structure. From the EFCs, if the phase velocity of the light wave is opposite to the energy flow, the Poynting vector $S$ and Wave vector $\mathrm{k}$ will be antiparallel, and negative refraction is realized. In 2002, Luo et al. studied the negative refraction of the cubic photonic crystal and simulated the image of a point source by using Finite-difference time-domain (FDTD) method [6]. Cubukcu et al. experimentally demonstrated the negative refraction and superlensing with $2 \mathrm{D} \mathrm{PhC}$ in the microwave region [7]. $\mathrm{Lu}$ et al. demonstrated an imaging experiment by using a slab of an effective-negative-index in the microwave region
[8]. Zhang xiangdong analyzed the relationship between the image resolution and the thickness of the PhC slab [9]. K. Aydin et al. realized the subwavelength resolution with the negative-index meta-material superlens [10]. Recently, annual photonic crystals (APCs) had attracted much attention due to the more adjustable parameters. Many promising properties have been found in APCs [11]-[15]. The imaging properties of an annual photonic crystal slab for TM and TE polarization have been analyzed by $\mathrm{H}$. Wu et al. [16].

In this paper, a two-dimensional (2D) hexagonal lattices annular photonic crystal (APC) was proposed. And the annular photonic crystal was obtained by introduced circular-airhole in the core of the Si-rod. The photonic band structures and equifrequent contours (EFCs) of the APCs with different radius of the circular hole were calculated by the Plane Wave Expansion (PWE) method. Negative refraction and subwavelength imaging of a point source are respectively demonstrated in the designed two-dimensional hexagonal lattices annular photonic crystal (APC) by the Finite-Difference TimeDomain (FDTD) method. At the same time, the spatial resolution of the annular photonic crystal superlens was analyzed in 


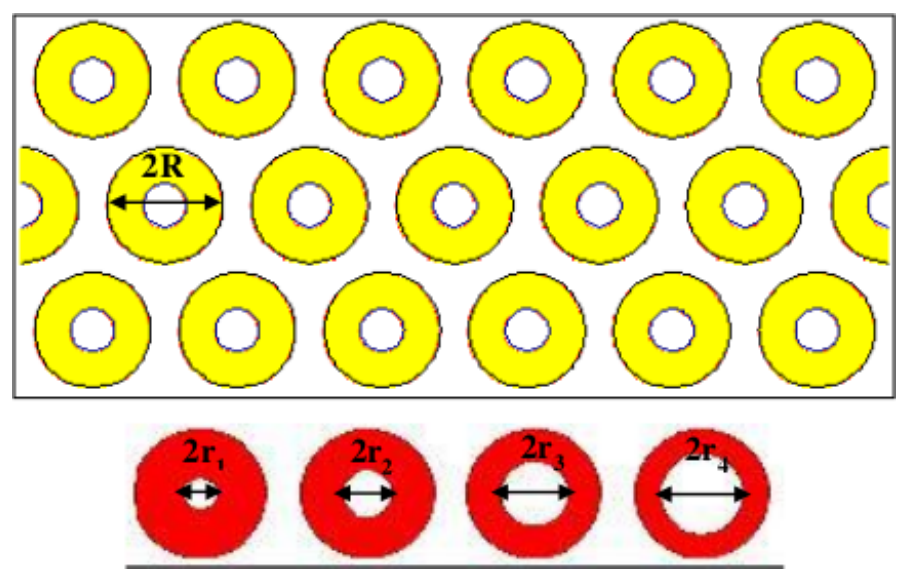

FIG. 1 Schematic of the hexagonal lattices annular photonic crystals (APCS), $R$ is the radius outer ring and $r$ is the inner cylinder radius. $R=0.4 a, r_{1}=0.1 a, r_{2}=0.15 a$, $r_{3}=0.2 a, r_{4}=0.25 a, \varepsilon=12.96$.

detail. It was also found that the resolution decreases linearly with the increasing of the inner radius.

\section{PROPERTIES OF THE DESIGNED APC WITH DIFFERENT INNER RADIUS}

The 2D hexagonal lattices annular photonic crystal (APC) with ring-shaped pillar as shown in Figure 1, the ring-shaped pillar was obtained by introduced circular-air-hole in the core of the Si-rod. Both the background and the core cylinder are airs, while the ring-shaped pillars material is chosen as silicon $(\varepsilon=12.96)$. The outer ring radius $R$ is $0.4 a$ and the inner ring radius $r$ is $0.1 a, 0.15 a, 0.2 a, 0.25 a$ respectively, where $a$ is the lattice constant. TM band structures of the designed APC with different $r$ were calculated by plane wave expansion method and they were shown in Figure 2. The normalized frequencies which realize superlense imaging can be obtained directly by finding the intersection point of the second band and the light line. From Figure 2, it can be found in the vicinity of normalized frequency $V_{g} \cdot \boldsymbol{k}<0$, negative refraction may occur around these frequencies. So we can get the normalized frequencies, which can realized negative refraction, $\omega_{1}=0.2983(2 \pi c / a), \omega_{2}=0.3064(2 \pi c / a)$, $\omega_{3}=0.3205(2 \pi c / a), \omega_{4}=0.347(2 \pi c / a)$ corresponding to different inner ring radiuses $r$. It can be concluded that the frequencies increases with the increasing of the inner radius.

It was known that the group velocity of the transmitted electromagnetic waves can be calculated from the equal frequency contours [17]. So the negative refraction can result from special shape EFCs of the designed APC. The frequencies in the lowest band may be more desirable in high-resolution superlensing. Taking into account the annular photonic crystal, we choose the inner ring radius $r$ is $0.1 a$, $0.15 a, 0.2 a, 0.25 a$ respectively. Figure 3 shows its EFCs computed over the first Brillouin zone for the second band, it can be found that the constant frequency curves, which consist of the allowed propagation modes for a specific frequency, are very nearly with the light line contour and their frequency decrease along the $\Gamma-\mathrm{M}$ and the $\Gamma-\mathrm{K}$. In the APC, the energy velocity vector coincides with the group velocity vector of

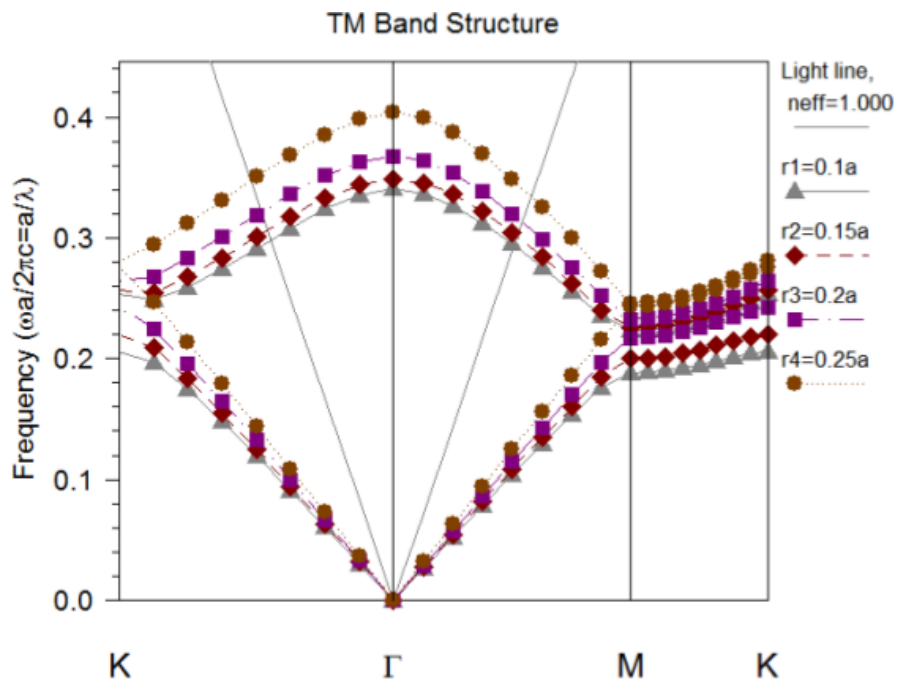

FIG. 2 TM band structure of the designed APC with $r=0.1 a, 0.15 a, 0.2 a, 0.25 a$ respectively. The dash line origin from $\Gamma$ represents light line.

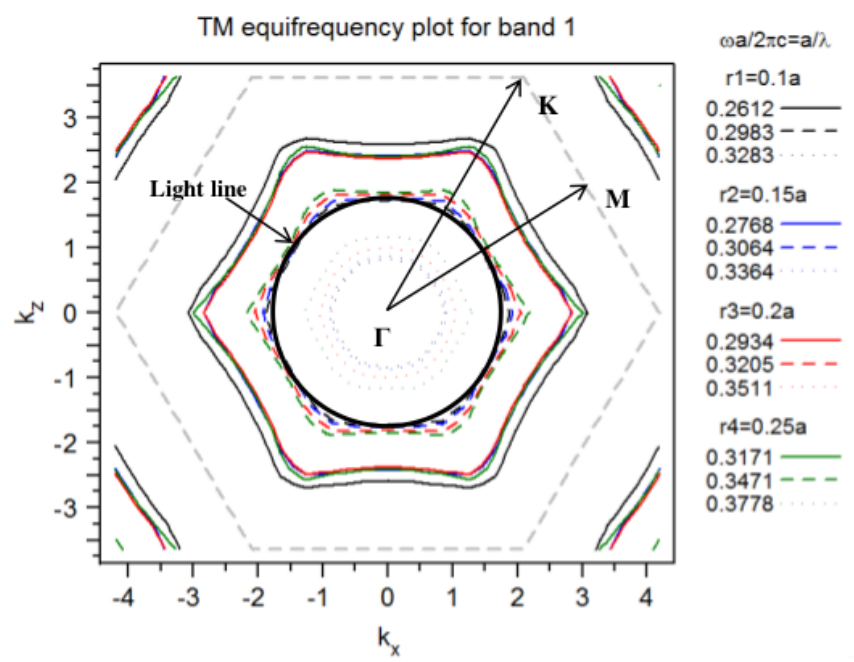

FIG. 3 The TM polarized band structure and the EFCS of the second band of the four 2-D hexagonal lattices APCs with different inner radius, frequency values are in units of $2 \pi c / a$. The frequency decreased along the $\Gamma-\mathrm{M}$ and the $\Gamma-\mathrm{K}$. The light line contour is very nearly with the second contour for the four APCs, implying the effective negative frequency $n_{\text {eff }}=-1$ can be found at the vicinal of this frequency.

the Bloch mode $v_{g}=\nabla_{k} \omega(k)$. This means that the group velocity is negative within this frequency range. So we can define an isotropic effective refractive index using Snells law. From Figure 2 and Figure 3, it can be observed that the frequency is $\omega_{1}=0.2983(2 \pi c / a), \omega_{2}=0.3064(2 \pi c / a)$, $\omega_{3}=0.3205(2 \pi c / a), \omega_{4}=0.347(2 \pi c / a)$ when effective refractive index is $n_{\mathrm{eff}}=-1$. It is well known that a left handed medium with $n=-1$ perfectly matches to free space, and therefore it should not reflects any incoming waves. Hence, the considered APC structure can leads to an unrestricted superlensing.

\section{SUPERLENSING IN APCS DEPENDING ON DIFFERENT INNER RADII}

Negative refraction effect can be used in many applications, such as absorber, filter, coupler, antenna, superlens, and superprism. So, the superlens can be realized in the designed 


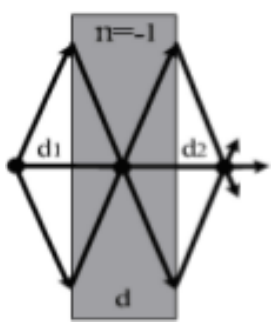

FIG. 4 Ray tracing illustration of the flat lens made of an isotropic homogeneous medium with index of refraction $d_{1}$ is the distance between the source and the first interface, and $d_{2}$ is the distance between the image point and the second interface, $d$ is the thickness of the slab.

APC (Figure 1) with effective-negative-index $n_{\mathrm{eff}}=-1$. In the case of a isotropic homogenous flat lens with $n=-1$, simple ray-optics introduce that the source-image distance remain constant and is equal to twice of the lens thickness as shown in Figure 4. There is no reflection at the interface between a negative index material of $n=-1$ and air. The coupling coefficient for any incident angle is always $100 \%$. Results for the APCs agreed fairly with this prediction. A continuouswave point source is placed at the distance of $3 a$ from the left surface of the four designed APC slabs. As we analyzed in the section 2, the frequencies which are corresponding to the case with $n_{\mathrm{eff}}=-1$ are $0.2983(2 \pi c / a), 0.3064(2 \pi c / a)$, $0.3205(2 \pi c / a), 0.347(2 \pi c / a)$ respectively. The field distribution of the point source and its image are simulated by FDTD method and plotted in Figure 5(a-d) with the inner radius $r=0.1 a, 0.15 a, 0.2 a, 0.25 a$ respectively. It can be found that a visible image spot formed at $2.89 a, 2.87 a, 2.81 a$ and $2.83 a$ from the right surface of the APC slabs with the reverse ordering for the corresponding inner radius. From Figure 5, it can be found, although small aberrations are visible in the field patterns, the imaging properties are as same as that of the isotropic homogeneous medium with refractive index $n=-1$. It means that the superlens imaging can happen in the designed APC systems at the range of normalized frequency from $0.2983(2 \pi c / a)$ to $0.347(2 \pi c / a)$. The aberrations are caused by the reflection at the interface between air and APC slab. The imaging point cannot perfectly couple with the source point. It is found that the coupling coefficient is highly angular dependent for an interface between air and a PhCs with $n_{\text {eff }}=-1$ [21].

\section{THE SPATIAL RESOLUTION OF THE APCS}

In order to analyze the spatial resolution of the APC, two continuous-wave point sources were placed at $3 a$ from the left surface of the APC slab. The electric field intensity distribution of the two point sources and its images were shown in Figure 6.

According to the Rayleigh Criterion, the spatial resolution of the designed APC slab is analyzed by reducing the distance between the two point sources. For the inner radius is $0.1 a$, the normalized frequency is $\omega=0.2983=a / \lambda$, as show in Figure $7(\mathrm{a})$, when the distance is $2.4 a$, the images could be dis-

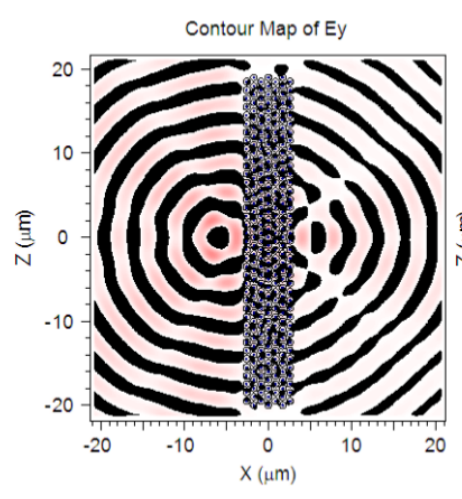

(a)

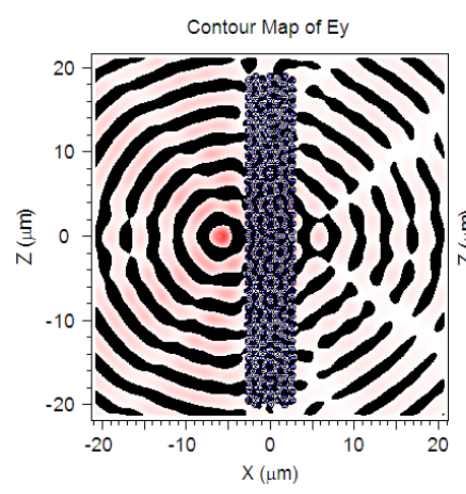

(c)

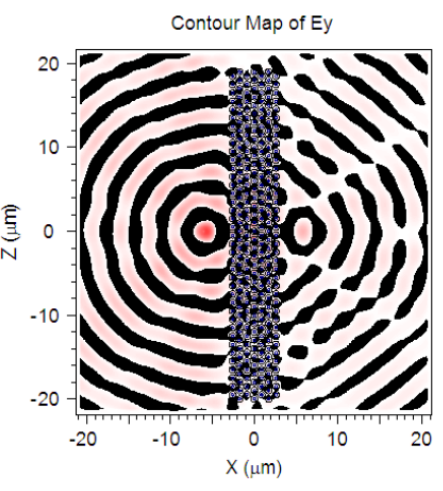

(b)

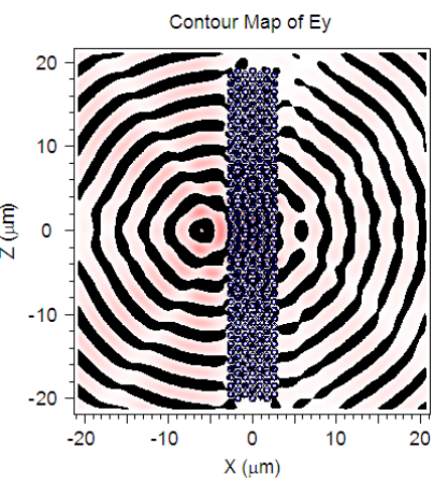

(d)
FIG. 5 FDTD simulation results of focusing a point source with a finite-width slab of $2 \mathrm{D}$ hexagonal lattices APCs for four different inner radii. The interface is perpendicular to the $\Gamma$-M direction. (a) $r_{1}=0.1 a, d_{2}=2.89 \mu \mathrm{m}$ and $\omega_{1}=0.2983(2 \pi c / a)$. (b) $r_{2}=0.15 a, d_{2}=2.87 \mu \mathrm{m}$ and $\omega_{2}=0.3064(2 \pi c / a)$. (c) $r_{3}=0.2 a, d_{2}=2.81 \mu \mathrm{m}$ and $\omega_{3}=0.3205(2 \pi c / a)$. (d) $r_{4}=0.25 a, d_{2}=2.83 \mu \mathrm{m}$ and $\omega_{4}=0.347(2 \pi c / a)$.

\begin{tabular}{llll}
$\begin{array}{l}\text { Inner } \\
\text { radius/a }\end{array}$ & $\begin{array}{l}\text { Normalized } \\
\text { frequency }\end{array}$ & Resolution/a & resolution/ $\lambda$ \\
\hline 0.1 & 0.2983 & 2.5 & 0.7458 \\
0.15 & 0.3064 & 2.3 & 0.7047 \\
0.2 & 0.3205 & 2.1 & 0.6731 \\
0.25 & 0.347 & 1.9 & 0.6593
\end{tabular}

TABLE 1 The resolution of the four designed APCS for different inner radius

tinguished obviously; when the distance of the sources is $2.5 a$, the images are just could be distinguished; when the distance is $2.6 a$, the images could not be distinguished. So, the subwavelength resolution is $2.5 a$, also is $0.7458 \lambda$. The other three different APCs have also been analyzed by this way, as shown in Figure 7(b-d). Table 1 is the simulation results of the four designed APCs. From it, we can conclude that the resolution decreases linearly with the increasing of the inner radius. But with the increase of the inner radius, the APC are more difficulty to produce and more fragile. So, the biggest inner radius was $2.5 a$. In the reference [16], $\mathrm{H}$. Wu et al. realized the image of an unpolarized wave point source in their designed APC slab and analyzed the image plane's intensity distribution for different the thickness of slab. In contrast, we analyzed the influence of the inner radius to the intensity distribution of the image plane and the resolution. 


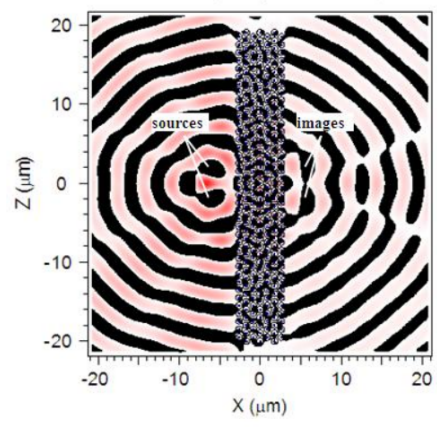

FIG. 6 The propagation distribution map of the APC with two point sources, two vertical point images were obtained in the right of the slab.

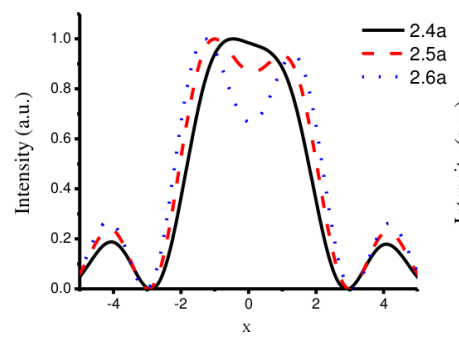

(a)

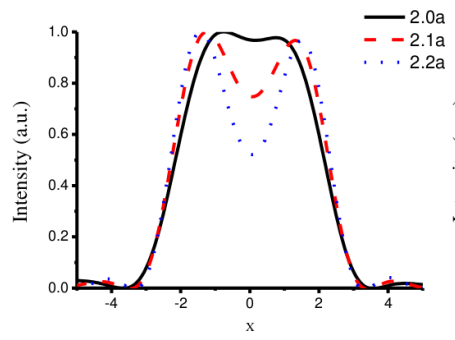

(c)

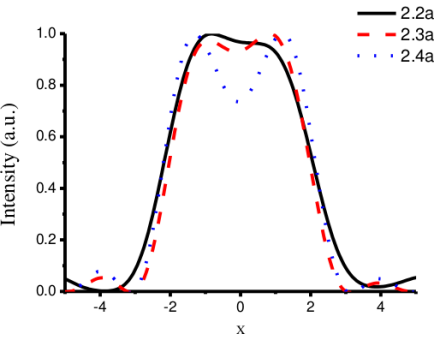

(b)

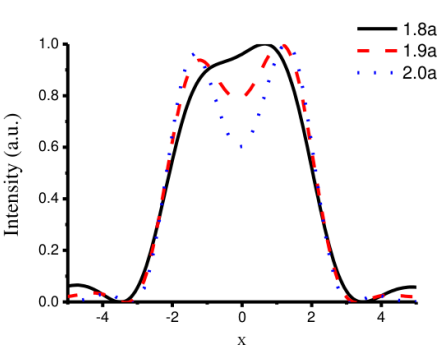

(d)
FIC. 7 Intensity of image of two point sources with different distances of sources for four different inner radii. The conclusion is the lager of the inner radius, the smaller of the resolution. (a) $r=0.1 a, d^{\prime}=2.5 a$, (b) $r=0.15 a, d^{\prime}=2.3 a$, (c) $r=0.2 a$, $d^{\prime}=2.1 a,(\mathrm{~d}) r=0.25 a, d^{\prime}=1.9 a$

\section{CONCLUSIONS}

In summary, the designed two-dimensional (2D) hexagonal lattices annular photonic crystal (APC) are studied by the plane wave expansion method and finite-difference time-domain method. Through the band structure, the equal-frequency contour and the numerical simulation, we investigate the negative refraction, superlensing effect and spatial resolution of the imaging which can be realized in the designed APC slab with the normalized frequency from $0.2983(2 \pi c / a)$ to $0.347(2 \pi c / a)$. It is found that the resolution decreased linearly with the inner radius increased. Actually, the APC has potential for optical components and would be applied to the integrated optical devices efficiently.

\section{ACKN OWLEDGEMENTS}

This work is supported by the National Natural Science Foundation of China (No.10904080, No.11144007, No.11274188), the Distinguished Middle-aged and Young Scientist Encourage and Reward Foundation of Shandong Province, China
(No. BS2011DX007) and supported by the State Key Laboratory of Transient Optics and Photonics (No. SKLST201012)

\section{References}

[1] E. Yablonovitch, "Inhibited Spontaneous Emission in Solid-State Physics and Electronics," Phys. Rev.Lett. 58, 2059 (1987).

[2] J. B. Pendry, "Negative Refraction Makes a Perfect Lens," Phys. Rev. Lett. 85, 3966 (2000).

[3] H. Kosaka, T. Kawashima, A. Tomita, M. Notomi, T. Tamamura, T. Sato, and S. Kawakami, "Superprism phenomena in photonic crystals," Phys. Rev. B 58, R10096 (1998).

[4] R. Moussa, S. Foteinopoulou, L. Zhang, G. Tuttle, K. Guven, E. Ozbay and C. M. Soukoulis, "Negative refraction and superlens behavior in a two-dimensional photonic crystal," Phys. Rev. B 71, 085106 (2005).

[5] A. Berrier, M. Mulot, M. Swillo, M. Qiu, L. Thyen, A. Talneau, and S. Anand, "Negative refraction at Infrared wavelengths in a twodimensional photonic crystal," Phys. Rev. Lett. 93, 073902 (2004).

[6] C. Luo, S. G. Johnson, and J. D. Joannopoulos, "All-angle negative refraction without negative effective index," Phys. Rev. B 65 , 201104 (2002).

[7] E. Cubukcu, K. Aydin, E. Ozbay, S. Foteinopoulou, and C. M. Soukoulis, "Negative refraction by photonic crystals," Nature 423, 604 (2003).

[8] J. Li, M.-H. Lu, T. Fan, X.-K. Liu, L. Feng, Y.-F. Tang, and Y.-F. Chen, "All-angle negative refraction imaging effect with complex two-dimensional hexagonal photonic crystals," J. Appl. Phys. 102, 7-073538 (2007).

[9] X. Zhang, "Image resolution depending on slab thickness and object distance in a two-dimensional photonic-crystal-based superlens," Phys. Rev. B 70, 195110 (2004).

[10] K. Aydin, I. Bulu, and E. Ozbay, "Subwavelength resolution with a negative-index metamaterial superlens," Appl. Phys. Lett. 90, 254102 (2007).

[11] H. Kurt, and D. S. Citrin, "Annular photonic crystals," Opt. Express 13, 10316 (2005).

[12] H. Kurt, R. Hao, Y. Chen, J. Feng, J. Blair, D. P. Gaillot, C. Summers, D. B. Citrin, and Z. Zhou, "Design of annular photonic crystal slabs," Opt. Lett. 33, 1614 (2008).

[13] A. Cicek, and B. Ulug, "Polarization-independent waveguiding with annular photonic crystals," Opt. Express. 17, 18381 (2009).

[14] J. Hou, D. S. Gao, H. M. Wu, and Z. P. Zhou, "Polarization insensitive self-collimation waveguide in square lattice annular photonic crystals," Opt. Comm. 282, 3172 (2009).

[15] L.-Y. Jiang, H. Wu, W. Jia, and X.-Y. Li, "Polarization-independent negative refraction effect in $\mathrm{SiO}_{2}$-GaAs annular photonic crystals," J. Appl. Phys. 111, 023508 (2012).

[16] H. Wu, L. Y. Jiang, W. Jia, and X. Y. Li, "Imaging properties of an annular photonic crystal slab for both TM-polarization and TEpolarization," J. Opt. 13, 095103 (2011).

[17] Z. Lu, C. Chen, C. A. Schuetz, S. Shi, J. A. Murakowski, G. J. Schneider, and D. W. Prather, "Subwavelength imaging by a flat cylindrical lens using optimized negative refraction," Appl. Phys. Lett. 87, 091907 (2005).

[18] P. Jiang, K. Xie, H. Yang, and Z. Wu, "Light propagation in two dimensional hexagonal lattices photonic crystal," J. Optoelectron. Adv. M. 13, 7 (2011). 
[19] Z. Ruan, Negative refraction and designed surface plasmons in periodic structures, (PhD Thesis, Microelectronics and Applied Physics Stockholm, Sweden, 2007).

[20] X. Wang, Z. F. Ren and K. Kempa, "Unrestricted superlensing in a triangular two dimensional photonic crystal," Opt. Express 12, 13 (2004).
[21] Y. Zhang, Y. Shen, J. Zhou, Y. Wang, F. Wu, J. Sun, and C. Guo, "Positive-negative birefraction phenomenon for TM polarization in annular photonic crystal," Chin. Opt. Lett. 9, 022601 (2011).

[22] X. Zhang, "Tunable non-near-field focus and imaging of an unpolarized electromagnetic wave," Phys. Rev. B 71, 235103 (2005). 